cells and the particles which are released show reduced infectivity. The latter phenomenon may be due to defective glycosylation of a virion protein. Interestingly, Friedman also reported that vesicular stomatitis virus produced by interferon-treated $\mathrm{L}$ cells is non-infectious, is deficient in G (glycoprotein) and $\mathbf{M}$ (matrix) protein content and appears abnormal in the electron microscope.

\section{Multiplying growth factors}

\section{from Denis Monard}

MORE knowledge about the events regulating the initiation of DNA synthesis will certainly shed more light on the phenomena regulating the differentiation of animal cells which remains one of the main challenges of modern biology. Such basic knowledge should also contribute to understanding better how and why a tumour cell differs from a normal cell.

Opening a recent discussion* on this topic R.W. Holley (Salk Institute, San Diego) re-emphasised that all growth is regulated by a multiplicity of complex extra and intracellular events. The discovery of proteins which can trigger cell growth, such as migration factor, epidermal growth factor (EGF), fibroblast growth factor (FGF) and platelet growth factor has revealed that extracellular macromolecules have an important role as regulators of the cell cycle, and the number of such factors known is steadily increasing.

Platelets have provided several new growth factors. The stimulation of cell growth by three polypeptides released by platelets has been reported (D. Paul, Temple University, Philadelphia). All three polypeptides cross-react immunologically and chromatograph under the same conditions in heparin Sepharose. They differ, however, in their molecular weight. One of them, a basic protein, is displaced by $\beta$-thyroglobulin or EGF. But, in contrast to growth stimulation by EGF, its biological activity is impaired by heparin. Another growth factor has been obtained from platelet extracts (W.J. Pledger, University of North Carolina, Chapel Hill) but analogies with the other growth factors released by platelets are premature.

Virus-transformed BHK fibroblasts also produce and release two proteins which stimulate the migration and DNA synthesis of normal fibroblasts (P. Leuthard, Friedrich Miescher-Institut, Basel).

A cell line with an especially large number of EGF receptors has been used to

-A round table discussion organised by $S$. Lacobelli and L. Jimenez de Asua on 'Regulation of initiation of DNA synthesis and differentiation in cultured animal cells' was held in Rome on 8 October, 1979 purify a sarcoma growth factor (SGF) After being fixed with formalin, these cells still bind the growth factor released by cells transformed by mouse sarcoma virus. The growth promoting activity was then eluted from the fixed monolayer by a $\mathrm{pH}$ change. A homogeneous preparation of SGF, biochemically and immunologically distinct from EGF, could be obtained after only two elution cycles. Interestingly, SGF and EGF differ in their biological activity. In contrast to EGF, SGF stimulates the normal fibroblasts to assume a 'transformed' morphology and to form colonies in semi-solid medium, a characteristic attributed only to tumour cells (G.J. Todaro, NIH, Bethesda). This elegant modification of affinity chromatography with fixed cells should certainly allow the discovery of other proteins able to trigger the initiation of DNA synthesis.

It is possible at this stage to distinguish two classes of cell growth promoting proteins, that is, those released by normal cells which could be considered as the normal growth regulators and those released by tumour cells or by virus-transformed cells. More detailed biochemical and immunological analyses are now required to bring some order into the classification of such macromolecular growth factors.

In addition, greater knowledge of their mode of action would allow a better classification. However, the following facts reported at this round table discussion illustrate the complexity of such studies.

For example, growth-stimulating molecules such as prostaglandin $\mathrm{F}_{2 \alpha}$ and FGF can act synergistically in $3 \mathrm{~T} 3$ cells $(\mathrm{V}$. Richmond, Friedrich Miescher-Institute, Basel).

Cells also release high and low molecular weight factors which stop the cell cycle and, therefore might be able to antagonise or alter the biological effects of the growth factors. For example, a macromolecule has been isolated which reportedly arrests cells in $G_{1}$, an effect also produced by $A C T H$, thyrotropin or interferon (Holley).

Culture conditions also indirectly influence the sensitivity of cells to growth factors. For example, the number of receptors for EGF drops with increasing cell density and the binding kinetics are also affected (Holley). Moreover, viral transformation of cells can lead to a loss of EGF receptors (Todaro). It is also important to remember that cell growth regulation in vitro can be influenced by physical parameters which are not exactly similar to those predominant in vivo. For instance, the type of collagen used to coat Petri dishes can modify cell sensitivity to EGF and cell survival (W.R. Kidwell, NIH, Bethesda).

However, the macromolecular factors are apparently not solely responsible for the regulation of the initiation of DNA synthesis. Low molecular weight substances such as the classical hormones and nutrients (prostaglandin $F_{2 \alpha}$, glucose, inorganic salts and so on) are also able to potentiate the effects of macromolecules or to elicit growth alone (Richmond; Holley). The field is now entering a phase in which one might consider as a working hypothesis, the existence of metabolic steps which are simultaneously triggered by all these different substances to initiate cell division. Nevertheless, it remains possible that the regulation of cell growth is due to a delicate balance between many chains of events which are not all metabolically linked.

Denis Monard is at the Friedrich MiescherInstitut in Basel.

\section{Tokamaks still to the fore}

\section{from R.J. Bickerton}

AT the 9th European Physical Society Conference on Controlled Fusion and Plasma Physics* the tokamak continued to hold the centre of the stage. Tokamaks are toroidal discharges maintained to a satisfactory level of stability by the superposition of relatively strong toroidal fields. The system has many advantages not least the simplicity and ease with which hot, well-confined plasmas can be obtained. It has however two serious disadvantages. Powerful additional heating is needed to get high temperatures and the ratio of plasma pressure to magnetic field pressure is usually very low. The heating requirement has been met in the past with outstanding success by injecting powerful beams of fast $(\sim 40$ $\mathrm{keV}$ ) neutral atoms. Record ion temperatures of the order $6 \mathrm{keV}$ were achieved in this way last year with more than $2 \mathrm{MW}$ of injected beam into the Princeton tokamak PLT. But the longer term prospects for this technique are dim because for reactor scale plasmas higher energy beams are needed, making it technically difficult, expensive and inefficient. Thus great attention was given at the meeting to the new results on radiofrequency heating. In one set of experiments a few hundred kilowatts of power at the frequency of ion cyclotron resonance were injected into the tokamaks TFR (Paris) and PLT. In both cases the ion temperature was increased by several hundred electron volts with an efficiency comparable to that of low energy neutral injection. In this frequency range (20-60 $\mathrm{MHz}$ ) there is no technical problem in generating the power; the main difficulty is in coupling the power to the plasma. Heating of tokamak plasmas at other plasma resonance frequencies was also reported, the most notable being the use of

*Held in Oxford on 17-21 September, 1979. 